\title{
Characteristics of chaotic processes in electrocardiographically identified ventricular arrhythmia
}

\author{
Andrzej Mysiak ${ }^{1}$, Małgorzata Kobusiak-Prokopowicz ${ }^{1}$, \\ Konrad Kaaz ${ }^{1}$, Kamila Jarczewska ${ }^{2}$, Wojciech Glabisz ${ }^{2}$
}

${ }^{1}$ Chair and Department of Cardiology, Wroclaw Medical University, Wroclaw, Poland ${ }^{2}$ Institute of Civil Engineering, Wroclaw University of Technology, Wroclaw, Poland

\begin{abstract}
Background: The theory of chaos proves a deterministic mechanism of induction of multiple complex processes previously thought to be random in nature. This research explains how these complex processes develop. The aim of the study was to test the hypothesis of the chaotic nature of myocardial electrical events during ventricular tachycardia (VT) and ventricular fibrillation (VF).

Methods: Original hardware and software was developed for digitalization of on-line electrocardiography (ECG) data, with the functions of automatic and manual identification as well as categorization of specific ventricular arrhythmias. Patient ECGs were recorded by specially developed measuring equipment (M2TT). Available ECG sampling frequency was 20,000 $\mathrm{Hz}$, and it was possible to analyze the signal retrospectively. Digital ECG of the sinus rhythm (SR) was analyzed with non-sustained VT, $V T$ and VF. The signals were then subjected to mathematical analysis. Using wavelet analysis, signals carrying frequencies from various ranges were isolated from baseline and each of these isolated signals was subjected to Fourier transformation to check on differences in the Fourier power spectra of the analyzed VT and VF signals.

Results: Ventricular tachycardia identified based on ECG fulfills the criteria of a chaotic process, while no such properties were found for SR and VF. Information obtained by the ECG is used to record myocardial electrical signals, but they are not sufficient to differentiate between an advanced chaotic state and the process of linear expansion of electrical activation within the myocardium.

Conclusions: Electrophysiological study requires advanced methods to record the signal of myocardial electrical activity, as ECG is not sufficiently sensitive to identify the features of a chaotic process during VF. (Cardiol J 2017; 24, 2: 151-158)
\end{abstract}

Key words: theory of chaos, ventricular fibrillation, ventricular tachycardia, wavelet analysis, phase trajectories

\section{Introduction}

The theory of chaos proves a deterministic mechanism of induction of multiple complex processes previously thought to be random in nature and the theory also explains how these complex processes develop [1, 2]. Identification of chaos states and their qualitative and quantitative description are the subject of studies in many disciplines, particularly in technical sciences, medicine, biology, economy, and astronomy.

Development of ventricular fibrillation (VF) is a multi-step process. The initial step is an event that initiates ventricular tachycardia (VT), and the

Address for correspondence: Dr. Konrad Kaaz, Chair and Department of Cardiology, Wroclaw Medical University, ul. Borowska 213, 50-556 Wroclaw, Poland, tel: +48 71736 4200, fax: +48 71736 4209, e-mail: konrad.kaaz@gmail.com 
other step is degeneration of VT into VF. However, clinical studies with therapies targeted to reduce the number of VT events were not successful at preventing VF [3, 4]. Efforts to develop effective drug therapies and prevention of this phenomenon are currently targeted at attempts to determine genesis and electrophysiological characteristics of the process of VT degeneration into VF.

Established electrophysiological methods do not allow for identification of the events underlying transition from VT to VF. The theory of chaos is particularly suited for VF research as it describes this phenomenon in a comprehensive way and shows that a deterministic system may be characterized by an ability to develop complex processes. The possibility to predict whether a deterministic chaos will be present in a given system deserves attention. From a mathematical perspective, all nonlinear dynamic systems with more than two degrees of freedom may show chaos. The basic condition for chaos to occur is thus nonlinearity of equations describing the dynamics of a system.

Early studies on VF using electrocardiography (ECG) failed to identify this phenomenon as showing properties of a chaotic process. Only after additional information offered by spatial analysis was taken into consideration, did it become possible to show an organized electrical activity during VF, with accompanying attributes that indicated determinism [5].

Numerical analysis of chaotic phenomena is one of the basic elements of elucidation of their critical states. Qualitative methods to evaluate chaotic states include analysis of their phase portraits, Poincare maps, Fourier spectral analysis, and analysis of bifurcation diagrams. The basic quantitative method which allows definite conclusions regarding the actual state of the studied phenomenon is the analysis of Lapunov exponents, which usually requires long periods of follow-up if a time series is investigated [6, 7].

Wavelet analysis and wavelet packet analysis, due to a multiresolution nature of wavelet functions, have also become increasingly common tools for qualitative evaluation of chaotic states of oscillating systems in many areas of science [8-11].

The aim of the present study was to test the hypothesis of a chaotic nature of myocardial electrical events during VT and VF, categorized using conventional ECG.

\section{Methods}

In cooperation with the Wroclaw University of Technology, software was modified which al- lows the analysis of digital data using mathematical tools for identification of chaotic phenomena. Original hardware and software for digitalization of on-line ECG data was developed with the functions of automatic and manual identification as well as categorization of specific ventricular arrhythmias. The study was approved by a Bioethics Committee.

Patient ECGs were recorded by speciallydeveloped measuring equipment (M2TT) that allowed recording of three limb leads (I, II, III) and two precordial leads (Va and $\mathrm{Vb}$ ) using the conventional chest lead arrangement. Available ECG sampling frequency was $20,000 \mathrm{~Hz}$, and it was possible to analyze the signal retrospectively. Samples of VT and VF tracings were prepared, according to ACC/AHA/ESC 2006 guidelines [12]. The signals from leads I, III, and Va (second right intercostal space, conventional V1) were then subjected to mathematical analysis.

Using wavelet analysis, signals carrying frequencies from various ranges were isolated from baseline VT and VF signals and each of these isolated signals was subjected to Fourier transformation to check on differences in the Fourier power spectra of the analyzed VT and VF signals.

In addition, the distribution of wavelet expansion coefficients of the analyzed VT and VF signals was shown in appropriate frequency ranges, corresponding to particular steps of wavelet decomposition of the measured data $(\{1\},\{0,1\}$ etc. $)$ as a function of time. Areas of increasing darkness indicate a higher number of significant wavelet expansion coefficients of the measured signal, corresponding to a given frequency component appearing at the given time.

Wavelet analysis was performed on a number of measurement points, using the Mayer wavelet as the analyzing function and subjecting the measured data to five steps $(n=5)$ of wavelet decomposition.

In addition to Fourier spectral analysis of the evaluated signals, which is one of the approaches to qualitative chaos identification, the phenomenon was also identified based on graphs of phase portraits and Poincare cross-sections. A characteristic feature of phase portraits of a chaotic system is noted that the phase trajectory fills up the area of the phase space, running through nearly all of its points. Poincare cross-sections of chaotic systems take a form of so-called strange attractors, or sets of points towards which trajectories go asymptotically in the phase space.

Wavelet packet analysis was used to obtain derivatives of the analyzed signals to generate phase portraits and Poincare cross-sections. Taking Walsh 
functions as the base functions, high- and low-pass filter coefficients of the functions used, along with appropriate weights calculated using the method reported in Napiórkowska-Ałykow and Glabisz study [13], allowing appropriate values to be obtained for the derivatives of the analyzed signals.

In addition, we analyzed the distribution of the energy of the analyzed signals, defined as the sum of squares of wavelet expansion coefficients as a function of the number of coefficients taken into consideration, and sorted from the lowest to the highest absolute values [11]. Analysis of the signal energy in chaotic states is characterized by the fact that at a given level of energy, a higher number of wavelet expansion coefficients must be taken into account compared to non-chaotic states.

\section{Results}

Of 86 patients admitted to the Department of Cardiology due to ventricular arrhythmia or to evaluate implanted cardioverter-defibrillator (ICD) function, the final analysis included 20 patients whose digital ECG samples fulfilled our assumptions regarding the quality of the digital signal and the sampling frequency. Signals from another 4 patients were rejected due to a very high number of artifacts precluding analog evaluation of the cardiac rhythm. Ultimately, 16 patients provided 120 digital sequences of the sinus rhythm (SR), and 450 tracings of non-sustained VT (nsVT) of between 4 to 8 beats in three analyzed leads. In 1 patient, VT was recorded during cardiac arrest, with overall 3 relevant tracing fragments obtained. In 9 patients with an ICD, 12 digital VF tracings and 12 VT tracings were recorded during routine defibrillation threshold testing. In addition, due to difficulties with arriving at a clear ECG diagnosis (VF vs. VT) when evaluating an analog recording of three recorded fragments in 1 patient, relevant digital signal tracings were categorized as VT/VF. All the patients with ventricular arrythmia had ischemic cardiomyopathy. Polymorphic VT or ventricular flutter had not been registered. The patients were treated with beta-blocker (100\%), none of them had amiodarone.

Based on this analysis, it was found that the most pronounced differences between SR, VT, VF, and VT/VF signals were seen in the distribution of wavelet analysis coefficients, as confirmed by the results shown in Figure 1.

The graph of phase portraits is derived as $\mathrm{f}(\mathrm{i})$ $(j, k)$ where $i$ is the $i$-th derivative of the signal $f, j$ is the $j$-th block of coefficients which can be reconstructed $\mathrm{i}$-th signal derivative and $\mathrm{k}$ is the $\mathrm{k}$-th level of the decomposition of wavelet packet analysis.

\section{Analysis of sinus rhythm signals}

Sinus rhythm was shown to be characterized by the presence of a discrete distribution of the Fourier spectrum of the analyzed signals, which is a characteristic of non-chaotic processes (Fig. 2C). This was also confirmed by the wavelet analysis which showed that significant coefficients of wavelet approximation of the given signal were present in both high and low ranges of the evaluated signal frequency (Fig. 1C). As the phase trajectory for SR does not fill the phase space by running through nearly all its points, it may be concluded that the signal is not chaotic (Fig. 3). The results of the analysis did not depend on the source of data, i.e., specific ECG lead.

\section{Analysis of ventricular tachycardia signals}

The distribution of the most significant coefficients of wavelet expansion of the VT signal as function of time is homogeneous for the whole time range in a given frequency range (Fig. 1B), indicating signal features typical for the chaos phenomenon. A continuous frequency spectrum, which is characteristic for the chaos phenomenon, occurs in the VT signal (Fig. 2B). The degree of this phenomenon depends on the lead chosen, but its nature is preserved in all leads from which signals were obtained. Fourier analysis of VT signals from various frequency ranges is characterized by a continuous spectrum of the data power, indicating that data sequences show features of a chaotic state. VT signal derivatives obtained by packet analysis were also characterized by the presence of a continuous Fourier spectrum, which was more evident than in the Fourier analysis of the actual measured data (Fig. 4).

Phase portrait analyses and Poincare crosssections also indicate that VT signals show features of a chaos phenomenon. This was also confirmed by the analysis of the distribution of energy from the analyzed signals.

Ventricular tachycardia signals are characterized by the need to use a higher number of wavelet expansion coefficients in comparison to the number of VF signal expansion coefficients at the same level of signal energy (Fig. 5). This is a result of a higher regularity and consistency in the distribution of VT signal expansion coefficients values and it is a characteristic feature inherent in chaos states. 


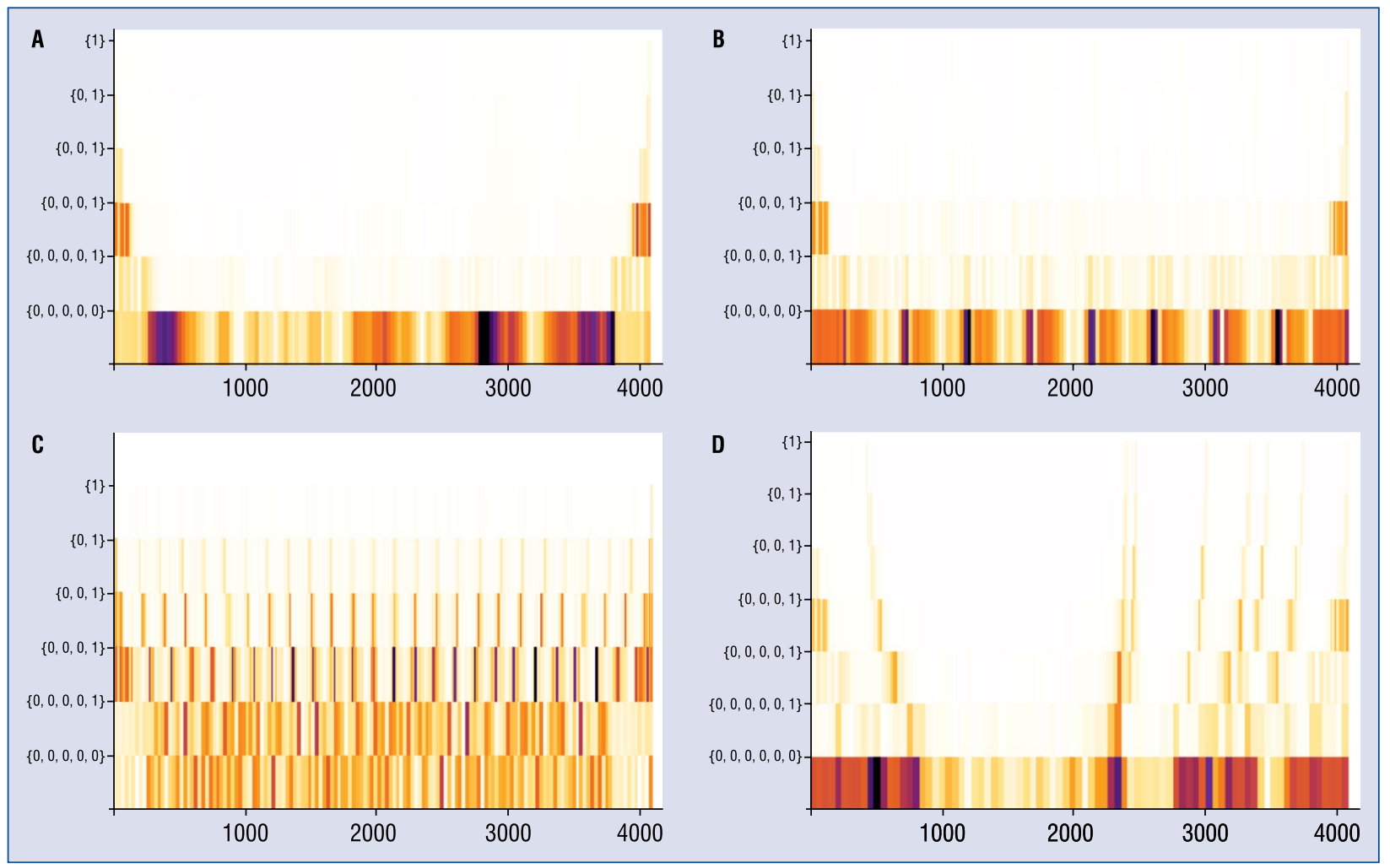

Figure 1. Distribution of wavelet analysis coefficients of the ventricular fibrillation (VF) (A), ventricular tachycardia (VT) (B), sinus rhythm (C), VT/VF (D) signals in lead I.

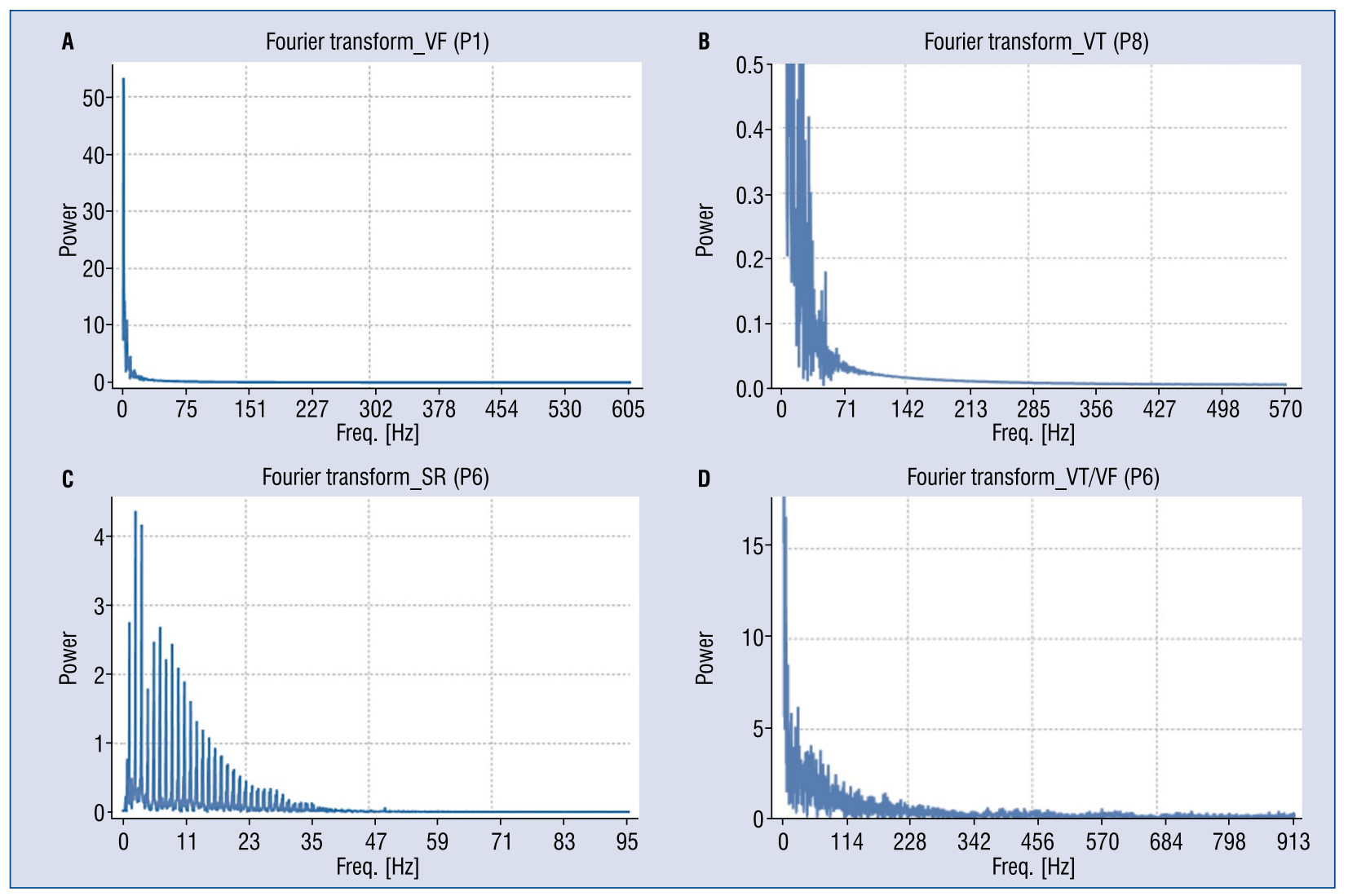

Figure 2. Power spectra (Fourier analysis) of ventricular fibrillation (VF) (A), ventricular tachycardia (VT) (B), sinus rhythm (SR) (C), VT/VF (D) signals in lead I. 


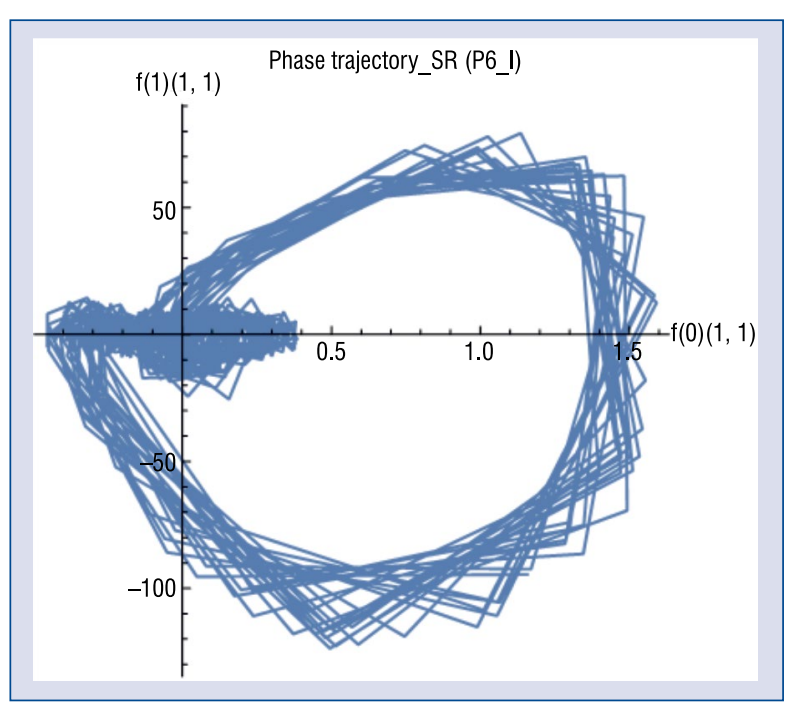

Figure 3. Phase trajectories of the sinus rhythm (SR) signal.

Phase function trajectories and Poincare crosssections of signals also show features indicating chaos; some of the presented phase functions largely fill the phase space, and Poincare crosssections show point sets towards which trajectories go asymptotically (Fig. 6).

\section{Analysis of ventricular fibrillation signals}

When analyzing signal fragments categorized as VF based on the ECG criteria, we found that the distribution of the most significant coefficients of wavelet expansion of the obtained signal (VF) as thefunction of time was not homogeneous for the whole time range in a given frequency range (Fig. 1A) (in contrast to the VT signal), indicating that the signal is not inherent in chaos phenomenon.

Fourier analysis of VF signals from various frequency ranges is characterized by a discrete spectrum of the data power, indicating that the signals show no features of a chaotic state (Fig. 2A). Similar findings were obtained regardless of the ECG lead used as the source of the analyzed signal (leads I, III, Va).

\section{Analysis of unclassified ventricular arrhythmia (VT/VF) signals}

For those analog recording fragments which could not be clearly categorized as VF or VT, qualitative methods of identifying a chaotic process proved insufficient to determine the nature of a digital signal described as VT/VF. It was also found that phase portraits in this case are characteristic for non-chaotic systems, Poincare cross-sections are characteristic for chaotic systems, and Fourier analysis did not allow definitive conclusions, showing features of both continuous and discrete distribution (Fig. 2D).

\section{Discussion}

Ventricular arrhythmias are a frequent mechanism of cardiac arrest and death in patients with

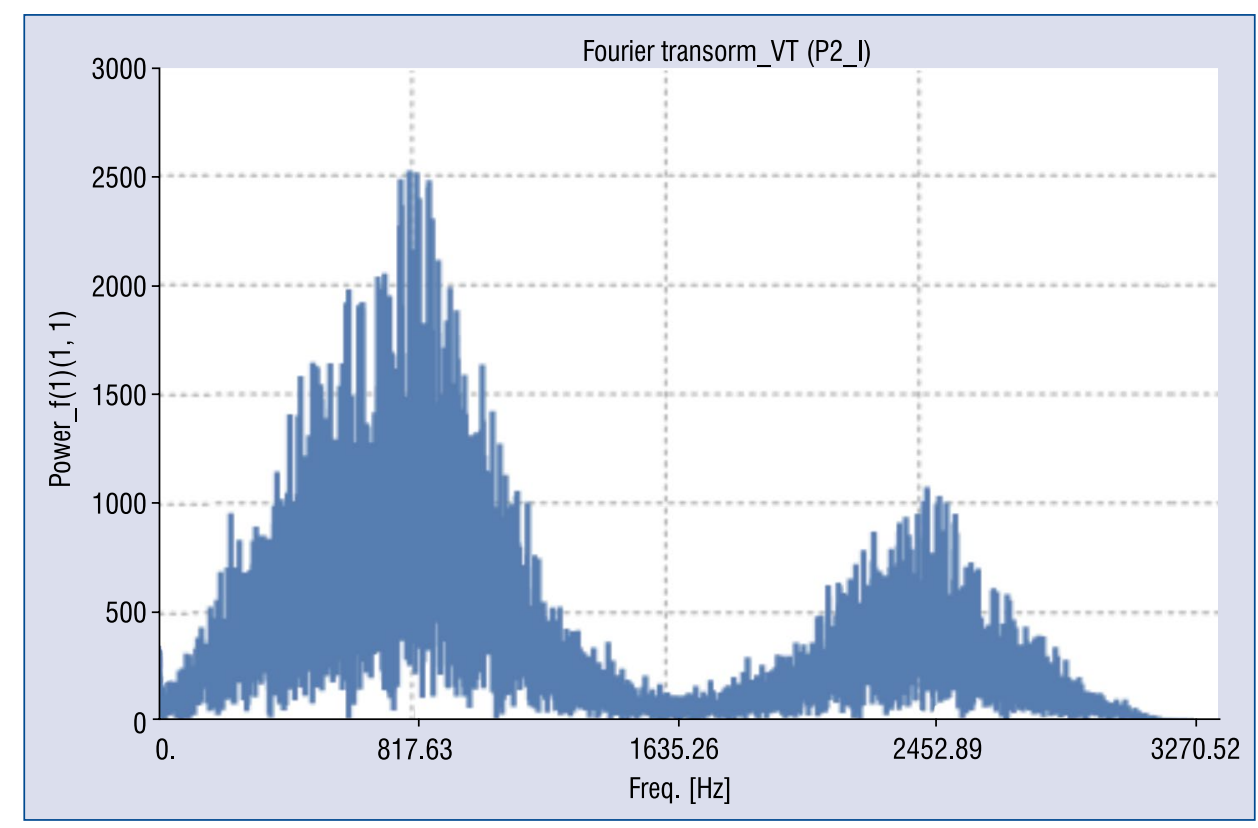

Figure 4. Fourier spectrum of the ventricular tachycardia (VT) signal derivatives $(f(i)(k, j))$ in lead I. Derivatives of VT signal were obtained using packet wavelets analysis. 


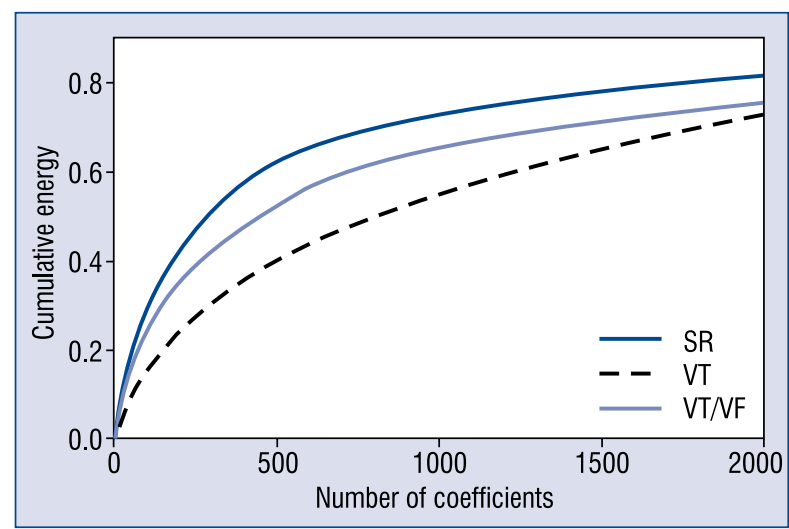

Figure 5. Distribution of the energy of ventricular tachycardia (VT) (dashed line), sinus rhythm (SR), VT/ventricular fibrillation (VT/VF) signals as a function of the number of wavelet expansions coefficient.

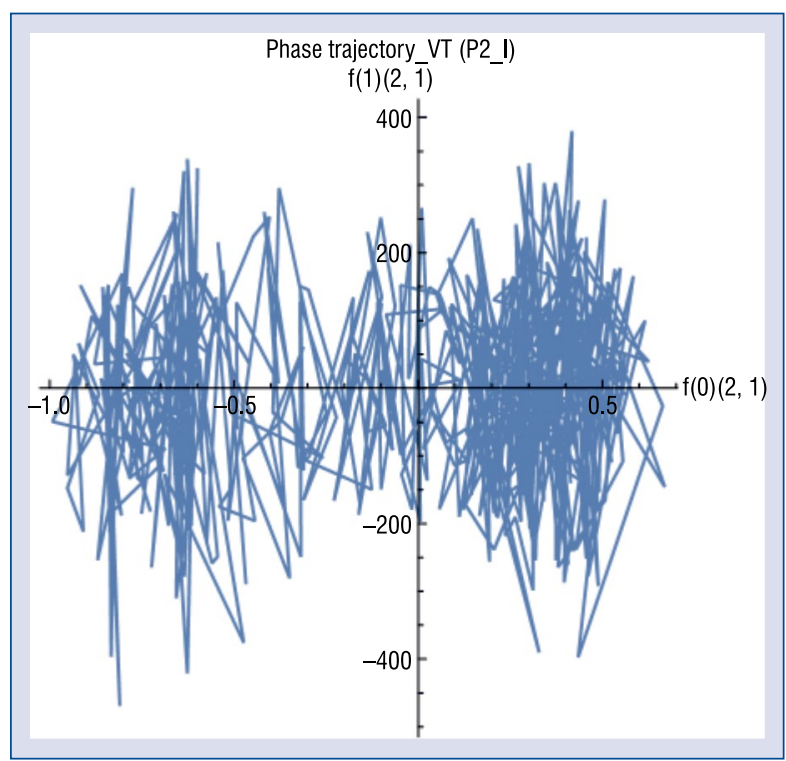

Figure 6. Phase trajectory of the ventricular tachycardia (VT) signal for patient P1.

acute and chronic cardiac disease. The occurrence of serious arrhythmia may be independent from the presence of risk factors, as it is associated with difficult-to-evaluate genetic predisposition and other factors modifying myocardial susceptibility to arrhythmia [14].

As indicated by experimental and clinical studies, the occurrence of VF is a consequence of previously initiated VT. Of note, however, as shown in the present study, is that categorization of malignant arrhythmia based on conventional ECG is sometimes difficult. Despite the established diagnostic value of ECG, recording of electrical events that occur rapidly in the myocardium, as in VT and VF, is associated with significant limitations of this diagnostic approach [15]. This is associated with insufficient sampling frequency of ECG, poor quality, and factors related to the analog form of the recorded signal. This was confirmed by an event of rapid ventricular arrhythmia recorded in one of the patients in the present study which could not be precisely diagnosed based on ECG morphology, and mathematical analysis of the digital signal was conducted using several approaches and did not allow explicit confirmation or exclusion of the chaotic nature of the arrhythmia.

In the present study, features of a chaotic process were noted in a sequence of signals obtained during electrocardiographically confirmed VT events. This is in agreement with previously published findings in an experimental model, showing the presence of chaotic characteristics during VT which then degenerates into VF, losing these properties [16]. When confronted with theoretical grounds, previous experimental studies and insufficient clinical data, however, it cannot be excluded that $\mathrm{VF}$ also has some features of a chaotic process but with advancement of its evolution to some degree, identification of deterministic characteristics at the level of data sampling frequency during ECG recording is no longer possible [1].

Results of large clinical trials indicate that efforts targeted exclusively at reducing ventricular arrhythmia in a high-risk patient population using available antiarrhythmic drugs, based on the arrhythmia suppression hypothesis, does not lead to a reduction in mortality [3]. Based on an analysis of computer models and several experimental studies, it was shown that some reported electrophysiological phenomena, categorized as ventricular arrhythmia, show features of a chaotic process. However, the present strategy of ICD implantation for primary and secondary prevention should be supplemented, based on identification of methods to pharmacologically reduce the risk of VT transformation into VF [17]. Another key aspect is to elucidate the nature of ventricular arrhythmia by defining the properties of those which show features of a chaotic process immediately after their initiation, which later evolves into VF. It has been assumed that a change in the duration and characteristics of an action may potentialy prevent spreading and breaking of spiral electrical waves within the myocardium, thus also preventing a potential event sequence culminating in VF. Such an approach may open new directions in the 
research on drug therapy in the treatment of ventricular arrhythmia.

In many previous studies, features of a chaotic state determined by a previous state were associated with VT. Results of the present study and previous experimental studies show that ECG signals of established VF, in contrast to VT, do not fulfil the criteria of a chaotic process.

It should also be noted that the nature of an ECG description is the detection of data associated with specific lead locations on the body surface and a constant frequency of sampling signals of an active process of electrical waves spread in the myocardium which undergoes significant changes with regard to the parameters which characterize it. In experimental conditions, use of spatial analysis confirms features of a chaotic process for VT $[5,18]$. Theoretical and experimental workindicates that by using leads that record signals from local myocardial areas beyond the scope of ECG detection, make it possible to identify a chaotic state. This requires another stage, a reduction in the radii of spreading action waves to be achieved. Attempts are thus made to use alternate detection systems, including optical leads that permit recording from several thousand sites, allowing for identification of the properties of an ever-shrinking process associated with the breakage of circulating activation waves into smaller ones, with continual reductions of their radii [19-21]. It may be argued that appropriate recording precision during the dynamic evolution of electrical phenomena in the myocardium is a prerequisite for further analysis of a chaotic state. It may also be concluded that standard surface ECG data are not sufficiently sensitive for identification of features of a chaotic state that is present during VF. This is a consequence of the evolution of chaotic dynamics [16]. In a synthetic assessment based on surface ECG signals, a set of electrical waves that reduce their spin diameter and break into smaller spinning structures after another critical curvature has been overstepped may form a new, non-chaotic state. Other data also indicate that a set of multiple concurrent chaotic process does not fulfil the criteria of a chaotic deterministic process [6, 7].

As shown in the present study, by using surface ECG to evaluate cardiac electrical activity during SR, VT, and VF, features of a chaotic process were shown only during VT. In the context of previous theoretical and experimental work, it cannot be excluded that VF also has some characteristics of a chaotic process, but the degree of its advancement does not allow the identification of features of a deterministic chaos at the level of ECG recording.

\section{Conclusions}

1. Ventricular tachycardia identified based on ECG recording fulfills the criteria of a chaotic process, while no such properties were found for SR and VF signals.

2. Information obtained by the ECG methods used to record myocardial electrical signal is not sufficient to differentiate between an advanced chaotic state and the process of linear expansion of electrical activation within the myocardium.

3. Electrophysiological study of the nature of chaotic process requires advanced methods to record the signal of myocardial electrical activity, as conventional ECG is not sufficiently sensitive to identify the features of a chaotic process during VF.

Funding sources: This research has been a part of the statutory activity of the Cardiology Department, Medical University of Wroclaw, Poland.

\section{Conflict of interest: None declared}

\section{References}

1. Weiss JN, Garfinkel A, Karagueuzian HS, et al. Chaos and the transition to ventricular fibrillation: a new approach to antiarrhythmic drug evaluation. Circulation. 1999; 99(21): 2819-2826, indexed in Pubmed: 10351978.

2. Witkowski FX, Kavanagh KM, Penkoske PA, et al. Evidence for determinism in ventricular fibrillation. Phys Rev Lett. 1995; 75(6): 1230-1233, doi: 10.1103/PhysRevLett.75.1230, indexed in Pubmed: 10060238.

3. The Cardiac Arrhythmia Suppression Trial (CAST) Investigators. Effect of encainide and flecainide on mortality in a randomized trial of arrhythmia suppression after myocardial infarction. N Engl J Med. 1989; 321(6): 406-412, doi: 10.1056/ /NEJM198908103210629, indexed in Pubmed: 2473403.

4. Waldo AL, Camm AJ, deRuyter H, et al. Effect of d-sotalol on mortality in patients with left ventricular dysfunction after recent and remote myocardial infarction. The SWORD Investigators. Survival With Oral d-Sotalol. Lancet. 1996; 348(9019): 7-12, indexed in Pubmed: 8691967.

5. Damle RS, Kanaan NM, Robinson NS, et al. Spatial and temporal linking of epicardial activation directions during ventricular fibrillation in dogs. Evidence for underlying organization. Circulation. 1992; 86(5): 1547-1558, indexed in Pubmed: 1423968.

6. Resnikoff HL, Wells RO. eds. One-Dimensional Wavelet Systems. In: Wavelet analysis. The scalable structure of information. Spinger-Verlag, New York 1998: 86-111.

7. Glabisz W. Analysis of wavelets in chaotic states for a onedegree-of-freedom system with viscoelastic restraints. J Sound Vib. 2002; 258(4): 633-648, doi: 10.1006/jsvi.2002.5142.

8. Permann D, Hamilton I. Wavelet analysis of time series for the Duffing oscillator: The detection of order within chaos. Phys 
Rev Lett. 1992; 69(18): 2607-2610, doi: 10.1103/PhysRevLett.69.2607, indexed in Pubmed: 10046538.

9. Staszewski WJ, Worden K. eds. The analysis of chaotic behaviour using fractal and wavelet theory. Proceedings of the International on Nonlinearity, Bifurcation and Chaos, Poland Lodz.; 1996: 222-226.

10. Billings SA, Coca D. Discrete Wavelet models for identification and Qualitive analysis of chaotic systems. Int J Bifurcation Chaos. 1999; 9(7): 1263-1284, doi: 10.1142/s0218127499000894.

11. Glabisz W. ed. Wavelet Analysis of Chaotic Signals. In: Packet wavelet analysis in mechanics problems (in Polish). Wroclaw University of Technology Publishing House. ; 2004: 33-49.

12. Zipes DP, Camm AJ, Borggrefe M, et al. American College of Cardiology/American Heart Association Task Force, European Society of Cardiology Committee for Practice Guidelines, European Heart Rhythm Association and the Heart Rhythm Society. ACC/AHA/ESC 2006 guidelines for management of patients with ventricular arrhythmias and the prevention of sudden cardiac death--executive summary: A report of the American College of Cardiology/American Heart Association Task Force and the European Society of Cardiology Committee for Practice Guidelines (Writing Committee to Develop Guidelines for Management of Patients with Ventricular Arrhythmias and the Prevention of Sudden Cardiac Death) Developed in collaboration with the European Heart Rhythm Association and the Heart Rhythm Society. Eur Heart J. 2006; 27(17): 2099-2140, doi: 10.1093/ eurhearti/ehl199, indexed in Pubmed: 16923744.

13. Napiórkowska-Alykow M, Glabisz W. Parametric identification procedure based on the Walsh wavelet packet approach for estimation of signal function derivatives. Arch Civ Mech Eng. 2005; 5: 5-26.

14. Morita N, Sovari AA, Xie Y, et al. Increased susceptibility of aged hearts to ventricular fibrillation during oxidative stress.
Am J Physiol Heart Circ Physiol. 2009; 297(5): H1594H1605, doi: 10.1152/ajpheart.00579.2009, indexed in Pubmed: 19767530.

15. Krstacic G, Krstacic A, Smalcelj A, et al. The „Chaos Theory” and nonlinear dynamics in heart rate variability analysis: does it work in short-time series in patients with coronary heart disease? Ann Noninvasive Electrocardiol. 2007; 12(2): 130136, doi: 10.1111/j.1542-474X.2007.00151.x, indexed in Pubmed: 17593181.

16. Mysiak A, Glabisz W, Kübler A, et al. Wykorzystanie teorii chaosu w badaniu elektrofizjologii migotania komór serca. Med Intens Ratunk. 2004; 7: 179-186.

17. Xie Y, Grandi E, Bers DM, et al. How does $\beta$-adrenergic signalling affect the transitions from ventricular tachycardia to ventricular fibrillation? Europace. 2014; 16(3): 452-457, doi: 10.1093/ /europace/eut412, indexed in Pubmed: 24569900.

18. Kaplan DT, Cohen RJ. Is fibrillation chaos? Circulation Res. 1990; 67(4): 886-892, doi: 10.1161/01.res.67.4.886.

19. Allesie MA, Bonke F, Schopman F. Circus Movement in Rabbit Atrial Muscle as a Mechanism of Tachycardia. Circulation Res. 1973; 33(1): 54-62, doi: 10.1161/01.res.33.1.54.

20. Dai S, Schaeffer DG. Chaos for cardiac arrhythmias through a one-dimensional modulation equation for alternans. Chaos. 2010; 20(2): 023131, doi: 10.1063/1.3456058, indexed in Pubmed: 20590327.

21. Witkowski FX, Leon LJ, Penkoske PA, et al. A method for visualization of ventricular fibrillation: Design of a cooled fiberoptically coupled image intensified CCD data acquisition system incorporating wavelet shrinkage based adaptive filtering. Chaos. 1998; 8(1): 94-102, doi: 10.1063/1.166290, indexed in Pubmed: 12779713. 